\title{
YOUR CONTRIBUTION TO THE LIBYAN DENTAL JOURNAL IS ANOTHER PIECE OF MOSAIC TOWARDS COMPLETING THE ENTIRE ORAL HEALTH IMAGE!
}

\author{
Kamis Gaballah', Mahmoud Elbashti² \\ 1) Department of Oral and Maxillofacial Surgery, Ajman University, Ajman, UAE. \\ Editor-in-Chief, The Libyan Dental Journal. \\ 2) Department of Maxillofacial Prosthetics, Graduate School, Tokyo Medical and Dental University, Tokyo, Japan. \\ Deputy Editor-in-Chief, The Libyan Dental Journal.
}

It has been seven years since the establishment of the Libyan Dental Journal (LDJ). During this period, we worked and still working towards the vision of improving the standards of scientific research and oral healthcare services nationally, regionally and extended to the rest developing countries. The journal aims to bridge the gap between oral health researchers, health policy makers and practicing dentists. It covers all major aspects of oral health research which might not appropriately covered by the international publications. We are totally aware of the increasing number of manuscripts being rejected by the international journals. This is largely due to the national and regional relevance that may not attract the attention of the readers of such periodicals. Other reasons for denying such manuscripts access to publication may include the novelty of the research, reliability of data, and inability of the international journal editors and reviewers to interpret the data from developing countries that unique to such population. Based on our awareness of the above mentioned matters, LDJ has worked from the beginning to have a harmonious mix of Libyan and international experts on various branches of oral health care from all over the globe. The editorial board also recruited many young Libyan researchers to expand their experience in all aspects of scientific publication including authorship, review and editorial ship at the highest standard possible.

Recently, many scientific activities related to dental sciences and oral health care have been taken place in many Libyan and regional institutions. Within these activities various high quality presentations were presented and shared, but for many of these research studies and case reports didn't get their way for publication. We believe that LDJ provides a suitable platform to all dental practitioners to publish their research studies and case reports. The LDJ accepts various types of manuscripts including Original Research, Continuous Education Reviews, Case Reports, Technical Reports, and Short Communications for publication. The submission is done electronically at the highest international standards and manuscripts will be reviewed by editors and the relevant expert reviewers. The accepted manuscripts will be published within a short time on the journal website and shall be available at all indexing links.

The LDJ editorial board is aware of the academic institutions demand and obligation of publication only in indexed and peer-reviewed platforms. In this regards, we confirm that every submitted manuscript is treated via several process to ensure that LDJ publish only the scientifically sound and guanine work that is carried out according to the best codes of practice in medical research. The manuscript-processing journey in LDJ starts with exclusion of plagiarized submissions through electronic software and side-by-side text comparison then the manuscript is assigned to an editor who will decide for the best reviewers of the work. If the outcome of review process is positive then the manuscript will pass through further 3 steps namely; copy-editing, layout-editing and proof reading to ensure the best form of the published paper.

Copyright $\odot$ 2018. LDJ. This is an open access article distributed under the Creative Commons Attribution 3.0 License, which permits unrestricted use, distribution, and reproduction in any medium, provided the original work is properly cited.

Citation:Libyan Dent J 2018, 8: 201812001 - http://dx.doi.org/10.5542/LDJ.v8i0.201812001 
The LDJ is currently preparing applications for its content to be indexed in the internationally reputable indices including Scopus and PubMed.

On behalf of the editorial board of the LDJ, we invite you to submit your valuable manuscripts at our website: http://academyih.org/journals/index.php/ldj/about/submissions. The online submission system will ensure a quick blind review and publication of your article. We also take this opportunity to call all colleagues and practicing dental practitioners, specialist dentists, dental technologists, academicians and researchers to support the LDJ by signing to our website as authors, readers or reviewers. In addition, it is highly recommended to subscribe to our e-alerts to get noted of any new articles published by LDJ. All these electronic services are provided free of charge by the publisher.

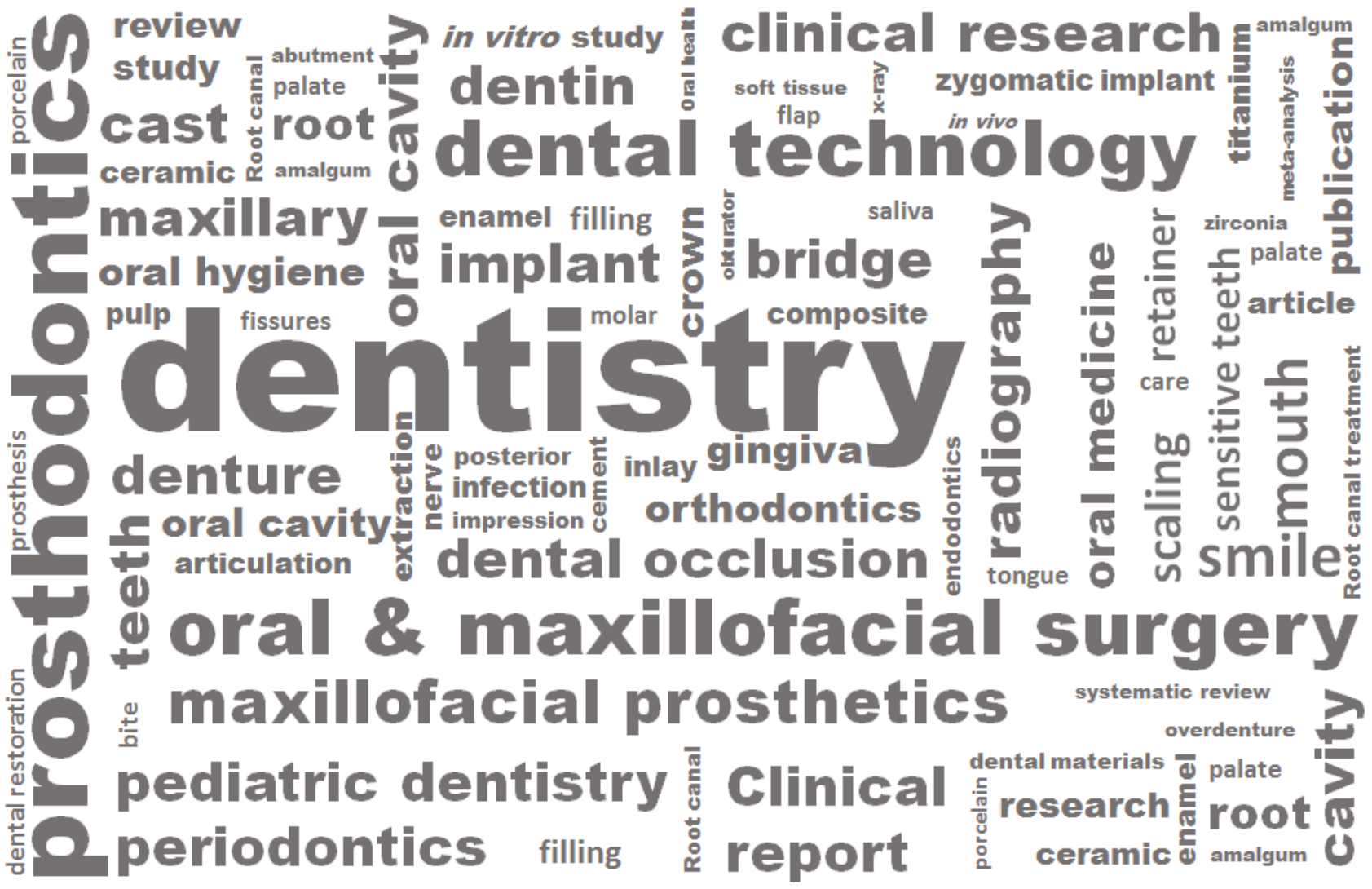

